\title{
A Modified Ant Colony Algorithm For Solving The Unit Commitment Problem
}

\author{
Ahmad Zand ${ }^{1}$, Mehdi Bigdeli ${ }^{1}$ and Davood Azizian ${ }^{2}$ \\ ${ }^{1}$ Department of Electrical Engineering, Zanjan Branch, \\ Islamic Azad University, Zanjan, Iran \\ ${ }^{2}$ Department of Electrical Engineering, Abhar Branch, \\ Islamic Azad University, Abhar, Iran
}

\begin{abstract}
Solving the unit commitment (UC) problem is one of the most complicated issues in power systems that its exact solving can be calculated by perfect counting of entire possible compounds of generative units. UC is equated as a nonlinear optimization with huge size. Purpose of solving this problem is to programming the optimization of the generative units to minimize the full action cost regarding problem constraints. In this article, a modified version of ant colony optimization (MACO) is introduced for solving the UC problem in a power system. ACO algorithm is a powerful optimization method which has the capability of fleeing from local minimums by performing flexible memory system. The efficiency of proposed method in two power system containing 4 and 10 generative units is indicated. Comparison of obtained results from the proposed method with results of the past well-known methods is a proof for suitability of performing the introduced algorithm in economic input and output of generative units.
\end{abstract}

\section{KEYWORDS}

Unit Commitment (UC), Nonlinear Optimization, Modified Ant Colony Optimization (MACO) Algorithm, Economic Dispatch (ED), Load Demand.

\section{INTRODUCTION}

The UC problem in power industry is a hard optimization problem which has enough potential for frugality of millions dollars in each year. In addition, it makes correct utilization of a network with on time units' inputs and outputs which it prevents quick exhaustion of instruments. The main purpose of UC problem is to minimize the cost of entire system utilization to determine situation of electrical energy units considering all constraints that supply the definite level of security. Actually, the issue of programming economic order of inputs and outputs for thermal units is an optimization problem which can be obtained by minimizing (or maximizing) the objective functions regarding all existed restrictions [1-3].

Using dynamic programming, the UC problem was first presented in 1966 by Lowery. Basically, the most careful method for solving this issue is the exhaustive enumeration. Hence, by analyzing all possible compounds of units in studied time-periods, considering all possible compounds is impossible (according to recent processor speed). So, necessity of employing a suitable algorithm seems to be urgent [4].

Used method for solving the UC problem can be divided to three parts: classical, smart and combinatory methods. For the first part, some methods can be pointed as exhaustive enumeration method, priority list, dynamic programming and Lagrange method, least square method [5]. These methods in terms of the isotropy and the response quality, these methods do not have high acceptability. The most common intelligent methods applied for solving this issue are taboo

DOI : 10.5121/aeij.2016.3302 
search [6-7], neural network [8], fuzzy logic [9], genetic algorithm [10-13], particle swarm optimization [14], and artificial bee colony algorithm [15-16], and teaching- learning based optimization [17].

Because of intricate calculations in input/output economic order programming of thermal units, practically a mathematical optimization is very hard to be developed for solving the UC problem precisely. The performing time of the problem will be increased based on the mathematical methods with exponential shapes. Since the number of operational constraints is increased, for keeping this time in a reasonable range, we should overlook many complicated constraints in problem formulization. Practically sometimes, it may cause a response to be unreachable. To solve this problem and for the purpose of omitting exhaustive enumeration method (which cannot be used as optimization result), a smart algorithm is used in this paper. Advantages of using expert systems for solving the thermal unit commitments, the input/output programming can be summarized as below:

1- Improvement of obtained results by regular actions which has been concluded during question and response council from operators and programming.

2- Considering some intricate performing constraints.

3 - Decreasing the time and the required computer memory.

4- Help new and inexperienced operators to appropriate planning

In recent years, the use of intelligent optimization methods to solve problems of power engineering has grown considerably [18]. The ACO introduced by Dorigo is one of best smart optimization algorithms [19]. The researchers [20-21] realized the capabilities of optimization by ant colony behaviors. In analysis moment, they understood that ants have ability of finding the shortest path to food. UC can be formulated as a conditional optimization problem and then, to solve it, a new version of ACO algorithm is used. For this purpose, a modified version of ACO algorithm has been developed using MATLAB software. Afterwards, it is employed in thermal units' production for two typical systems containing four and ten generators for supplying the load demand and the power system spinning reserve during eight and ten hours respectively. Initial ingredients have paid attention including the diary load demand's curve, spinning reserve, minimum stop time, start-up cost, stopping cost and thermal units' properties such as the minimum work time.

The proposed algorithm is able to gain the most optimized case by high speed in terms of economy for the units input and output order by considering the told constraints. Also it gains the amount of generative optimization for each unit (economic dispatch) which it's obtained results are better than the existed results in literatures.

\section{UC PROBLEM ForMulation}

Previously, UC problem has been solved using ACO [22-23]. In this study, a modified version of the ACO is proposed. So as to avoid prolongation of the paper, more well-known details about ACO will be not mentioned.

\subsection{Objective function}

For solving the UC problem, at first the cost function that is needed to be minimized, should be obtained. This cost function can be stated as follows [6]:

$C=\operatorname{Min} \sum_{t=1}^{T}\left\{\sum_{i=1}^{n}\left[c_{i}\left(P_{i}^{t}\right)+u_{i}^{t}\left(1-u_{i}^{t-1}\right) S_{i}\left(X_{i}^{t}\right)\right]\right\}$ 
Where:

$\mathrm{T}=$ Programmed period (usually 24 hours)

$\mathrm{N}=$ Number of programmed thermal units

$\mathrm{C}_{\mathrm{i}}\left(\mathrm{P}_{\mathrm{i}}^{\mathrm{t}}\right)=$ Production cost of $\mathrm{i}$-th unit

$\mathrm{P}_{\mathrm{i}}^{\mathrm{t}}=$ Production amount of $\mathrm{i}$-th unit in period of $\mathrm{i}$-th

$\mathrm{U}_{\mathrm{i}}^{\mathrm{t}}=$ Mood of $\mathrm{i}$-th unit in period of $\mathrm{t}$-th. (on unit Uit $=1$, off unit Uit $=0$ )

$X_{i}^{t}=$ Number of periods that $i$-th unit was Off

$\mathrm{S}_{\mathrm{i}}\left(\mathrm{X}_{\mathrm{i}}^{\mathrm{t}}\right)=$ Triggering cost function for $\mathrm{i}$-th unit

(In (1) each period usually is considered one hour).

\section{Rules}

In this paper the objective function (1) has been routed using the proposed ACO based algorithm and the rout with minimum cost has been gained. For employing the introduced algorithm, applying the following rules is essential:

\subsubsection{Pass rule or tie selection constraint}

Suppose that k-th ant is placed in $\mathrm{i}$-th point. In this case the possibility of $\mathrm{j}$-th point selection as destination and/or otherwise the selection of ij relocation path is obtained from equation (2) [8]:

$P_{i j}(k)(t)=\left\{\begin{array}{c}\frac{\left[\tau_{i j}(t)\right]^{\alpha} \cdot\left[\frac{1}{L_{i j}}\right]^{\beta}}{\sum\left[\tau_{i j}(t)\right]^{\alpha} \cdot\left[\frac{1}{L_{i j}}\right]^{\beta}} j, s \in N_{i}^{k} \\ 0\end{array}\right.$

Where:

$\tau_{\mathrm{ij}}(\mathrm{t})=$ Remained pheromone amount in ij relocation path in $\mathrm{t}$ time.

$\mathbf{L}_{\mathrm{ji}}=$ A amount that indicates ij relocation path acceptability rate.

$\mathrm{N}_{\mathrm{i}}{ }^{\mathrm{k}}=$ Set of points which $\mathrm{i}$ is progressed.

$\alpha=$ A parameter which determines pheromone weight effect on algorithm.

$\beta=$ A parameter which determines acceptability weight effect on algorithm.

\subsubsection{Pheromone Matrix}

In applying ACO algorithm we will need a common memory. This common memory is earned by generation of a pheromone matrix. For each path that can be selected by ant, some initial pheromone is considered and by selecting that path, some pheromone is added to the existing pheromone. Updating the local pheromone is operated as like as the following:

After each ant chooses the next destination, it updates the pheromone according to (3) [23].

$$
\tau_{i j}=(1-\rho) \tau_{i j}+\sum \Delta \tau_{i j}
$$

Where, $p$ is a coefficient between $[0,1]$ and one which is related to evaporation of pheromone in each path, and $\Delta \tau$ is considered as increasing amount of pheromone in the path.

\subsubsection{Stop Criterion}

If the number of ants passing a path get more than quorum and /or it have a heavy difference with the number of pass from other paths and the cost of that path become less, It can be concluded 
that the algorithm has achieve the optimal answer. It should be noticed that if none of upmentioned constraints do not come true the problem is not optimized.

\section{Proposed METhod For SOlVING UC Problem}

In past researches [22-23], UC problem is resolved by the ACO. In this paper, a new version of ACO is introduced to solve the UC problem. The proposed method which is in fact caused by making some changes in the ACO algorithm is able to reach the most optimized answer. Among the differences of the proposed algorithm with the ACO algorithm, here we consider a pheromone matrix for each hours of the programming junction. So, for a system containing 4 generators, it considers totally 8 pheromone matrixes and for a system involving 10 generators, it shall consider 24 matrixes.

Whereas, as the size of system growths, number of cases passed by ants will be increased from the first to the last hour. Therefore, the number of states must be limited in order to achieve better results in a shorter time. For example in a system containing generators for 24 hours, each ant confronts $10^{\wedge} 24$ cases. The simplest way to decrease number of cases in the UC problem is to establishing the units' priority list. The way is done by calculating the average cost of utilization of each unit in full load; the units' priority list can be obtained as there is a priority between the one by less cost and high cost. But, for a system of 4 generators this list has is not used because of the large number of constraints.

The proposed algorithm in this paper, called as MACO (modified ACO). As the number of constrains in the proposed algorithm is more and as the problem conditions are different, the MACO has many differences with ACO [22-23]. These differences are mentioned in the following sub-sections.

\subsection{New constants}

PNEEDED: required generating power (consuming load) for this hour

UNITS: The number of generating power units.

PMAX: Maximum of each unit's generating power.

PMIN: Minimum of each unit's generating power.

ALPHA, BETA, GAMA: Variables of fuel cost function according to output power.

MU: Minimum hours which unit must remain (turning on) after turning on in circuit.

HSC: Start-up cost by hot method.

CSC: Start-up cost by cold method.

CSHRS: The number of hours that we should use the start-up by cold method.

INITIAL: The number of hours that machine was off or on.

STATE: The State of machine being on or off.

PDESIRED: Sum of generating power and required circle saving for this hour, where by considering $\% 10$ of circle saving will be $\% 110$ of generating power or $1.1 \times \mathrm{P}$ NEEDED.

\subsection{The number of samples in converting continuous period to discrete period}

Just like ACO, in MACO continuous periods should be divided to discrete periods that the number of these periods should be equal for all power units. But main difference between ACO and MACO is how to decretive the intervals. In MACO, discrete amounts must be chosen in a way that the possibility of power units' collection reaches to a specific amount such as $450 \mathrm{MW}$ (regarding the load supply constraint). For this purpose, the last unit must have a value between its minimum and maximum generation. Therefore the number of discrete quantities is considered 
against the minimum difference and the maximum rate of the last generation unit; for other units, the same number of discrete samples must be selected.

Whereas, for power station, the possibility of being off should be provided. In addition to generated discrete amounts, a zero has been added as units off case for each unit.

For unit=1: UNITS

graph(unit,:)=[0, floor (linspace (PMIN (unit), PMAX (unit), SAMPLES-1))];

End

\subsection{Roulette wheel with specific conditions}

One of the intricate parts of the introduced UC program is calculating the required amounts for roulette wheel. The roulette wheel must be implemented in such a way that each unit to be off or to generate between its minimum and maximum power and the total power generated by units be equal to this hour. For this purpose, before performing roulette wheel for each unit, the minimum and maximum of required power should be calculated. These amounts are different from unit's maximum and minimum power generation (Pmax and Pmin) that are calculated according to the rate of produced power by previous units up to now (p-now) and unit's minimum and maximum generated power after this unit.

By assuming that all the units are on after this unit, by reducing the generated power up to now and by sum of unit's minimum generated power after this unit, the maximum amount of required power in this hour can be obtained as.

p_u_needed_max $=$ PNEEDED-p_now-sum(PMIN(unit+1:end));

For the minimum power amount that is required to be generated by each unit, exactly the same way can be acted., The only difference is that the maximum power of unit is considered instead of the minimum amount:

p_u_needed_min=PNEEDED-p_now-sum (PMAX (unit +1 :end));

But this is possible that required minimum power is lower than unit's minimum generating power and maximum required power is higher than unit's maximum generating power. So, for avoiding the possibility of selecting out of unit's generating amount, between minimum required power and minimum generating power, we get maximum and between required maximum power and unit's maximum generating power we get minimum.

Now, for roulette wheel calculation, we should separate the required amounts (which are minimum and maximum among created discrete amounts). But up to now it did not discussed about units' off mode! This unit can be off while the required power be lower or equal to zero. In another case, while none of the generated discrete amounts are not obtained between minimum and maximum of the unit, this unit is forced to be off.

While we are at the first stage of required minimum and maximum power calculation, it is supposed that all of the units after this unit be on, but this is not always true and also the system's off mode should be considered. For this purpose, the required power at this moment should be lower than the maximum power of unit until the unit generates all required power (other units be off). By doing these calculations, necessary amounts have been prepared for performing roulette wheel; the remaining stages of roulette wheel are similar to ACO algorithm.

\subsection{Constraints}

Among existing constraints in UC, the constraints of minimum and maximum generated power for each unit and the load supply are considered directly for roulette wheel calculation. Economic dispatch (ED) constraint among units is problem's main goal and it will be intend to while minimizing the fitness function. Hence, three constraints remains that we mention them in below. 


\subsubsection{Minimum off-time constraint for each unit}

If a unit is off and we have not still achieved the minimum off-time, two modes are possible to occur:

1- Unit's off-mode possibility is provided which in this case it is informed that the unit is off and without doing roulette wheel; the next unit will be selected.

2. Unit's off-mode possibility is not provided which in this case the possibility of continuing ED is not provided and ED should be performed among the units for the related ant from the beginning of the optimization process.

\subsubsection{Minimum on-time constraint for each unit}

This constraint must be perused both before and after roulette wheel. Before roulette wheel implementation, we should peruse that if the unit must be on but the required maximum is lower than the minimum generated power and is forced to be off, the possibility of continuing ED is not provided for ant and ED should be performed among the units for the related ant from the beginning of the optimization process.

\subsubsection{The constraint of the system's spinning reserve}

After finishing ED for each ant among the units, we should peruse that whether the total maximum generated power of the active units in circuit is more than the total required power generation in the current time and the system's spinning reserve? While the answer is no, ED should be performed among the units for the current ant from the beginning of the optimization process.

\subsubsection{Fitness function}

For fitness calculation, the main equation for each unit's cost is as same as consuming fuel cost function at output power which its parameters are obtained using constants such as ALPHA, BETA, and GAMA. Moreover, in the unit's off mode during previous hours, we should add the start-up cost to one of the cold or hot methods. If the off mode time of the unit be more than its CSHRS, starting is done by cold method otherwise start-up will be done by hot method. After calculating costs for each unit, by collecting all of these amounts, fitness quantity are earned for all the units.

\section{SimUlation RESULTS AND DISCUSSION}

\subsection{Simulated system properties}

For simulating the required system for UC problem, two groups of information is needed:

1- The load demand curve

2- The unit's characteristics

Where, here the above information is brought for two systems by four and ten generators.

\subsubsection{Load demand curve}

As we explained in the previous sections, for solving this problem a curve is needed which determines the amount of load demand in every hour. So, in Figure 1, a load curve is introduced for 8 hours related to a system with 4 generators and Figure 2 shows this curve for 24 hours related to a system with 10 generators.

\subsubsection{Unit's characteristics}

The characteristics of the units which are used by 4 and 10 generator systems are shown in tables 1 and 2 respectively. 


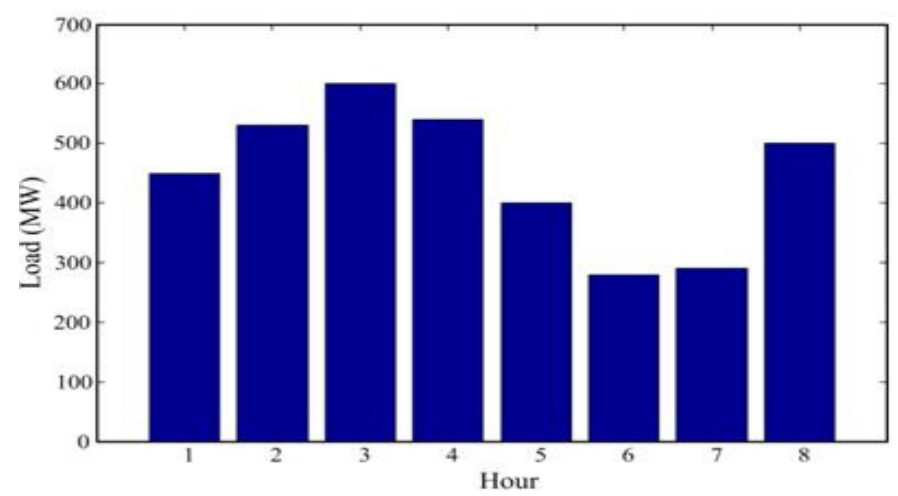

Figure 1. Load demand curve for a system by 4 generators

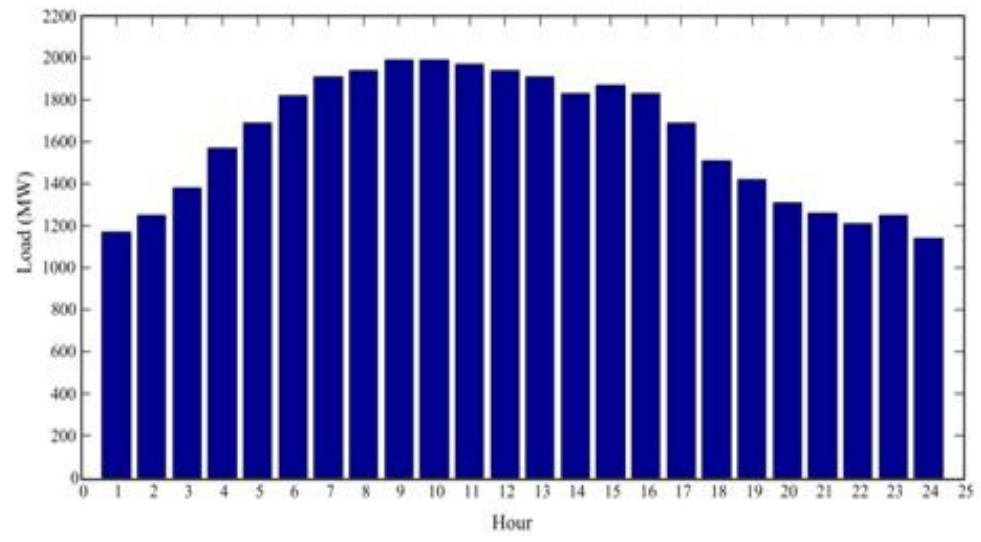

Figure 2. Load demand curve for a system by 10 generators

Table 1. Unit's properties in a sample system by 4 units

\begin{tabular}{|c|c|c|c|c|c|c|c|c|c|c|c|}
\hline unit & Pmax & Pmin & $\begin{array}{c}\boldsymbol{\alpha} \\
(\mathbf{S} / \mathbf{h})\end{array}$ & $\begin{array}{c}\boldsymbol{\beta} \\
(\mathbf{S} / \mathbf{M W h})\end{array}$ & $\begin{array}{c}\boldsymbol{\gamma} \\
\left(\mathbf{S} / \mathbf{M W}^{\mathbf{2}} \mathbf{h}\right)\end{array}$ & $\begin{array}{c}\text { MU } \\
(\mathbf{h})\end{array}$ & $\begin{array}{c}\text { MD } \\
(\mathbf{h})\end{array}$ & $\begin{array}{c}\text { HSC } \\
(\mathbf{S})\end{array}$ & $\begin{array}{c}\text { CSC } \\
(\mathbf{S})\end{array}$ & $\begin{array}{c}\text { Cs_hrs } \\
(\mathbf{h})\end{array}$ & $\begin{array}{c}\text { initial state } \\
(\mathbf{h})\end{array}$ \\
\hline 1 & 300 & 75 & 648.74 & 16.83 & 0.0021 & 5 & 4 & 500 & 1100 & 5 & 8 \\
\hline 2 & 250 & 60 & 585.62 & 16.95 & 0.0042 & 5 & 3 & 170 & 400 & 5 & 8 \\
\hline 3 & 80 & 25 & 213.00 & 20.74 & 0.0018 & 4 & 2 & 150 & 350 & 4 & -5 \\
\hline 4 & 60 & 20 & 252.00 & 23.60 & 0.0034 & 1 & 1 & 0 & 0.02 & 0 & -6 \\
\hline
\end{tabular}

Table 2. Unit's properties in a sample system by 10 units

\begin{tabular}{|c|c|c|c|c|c|c|c|c|c|c|c|}
\hline unit & Pmax & Pmin & $\begin{array}{c}\boldsymbol{\alpha} \\
(\mathbf{S} / \mathbf{h})\end{array}$ & $\begin{array}{c}\boldsymbol{\beta} \\
(\mathbf{S} / \mathbf{M W h})\end{array}$ & $\begin{array}{c}\boldsymbol{\gamma} \\
\left(\mathbf{S} / \mathbf{M W}^{2} \mathbf{h}\right)\end{array}$ & $\begin{array}{c}\text { MU } \\
(\mathbf{h})\end{array}$ & $\begin{array}{c}\text { MD } \\
(\mathbf{h})\end{array}$ & $\begin{array}{c}\text { HSC } \\
(\mathbf{S})\end{array}$ & $\begin{array}{c}\text { CSC } \\
(\mathbf{S})\end{array}$ & $\begin{array}{c}\text { Cs_hrs } \\
(\mathbf{h})\end{array}$ & $\begin{array}{c}\text { initial state } \\
(\mathbf{h})\end{array}$ \\
\hline 1 & 200 & 80 & 82 & 1.2136 & 0.00148 & 3 & 2 & 70 & 176 & 3 & 4 \\
\hline 2 & 320 & 120 & 49 & 1.2643 & 0.00289 & 4 & 2 & 74 & 178 & 4 & 5 \\
\hline 3 & 150 & 50 & 100 & 1.3285 & 0.00135 & 3 & 2 & 50 & 113 & 3 & 5 \\
\hline 4 & 520 & 250 & 105 & 1.3954 & 0.00127 & 5 & 3 & 110 & 267 & 5 & 7 \\
\hline 5 & 280 & 80 & 72 & 1.3500 & 0.00261 & 4 & 2 & 72 & 180 & 3 & 5 \\
\hline 6 & 150 & 50 & 29 & 1.5400 & 0.00212 & 3 & 2 & 40 & 113 & 2 & -3 \\
\hline 7 & 120 & 30 & 32 & 1.4000 & 0.00382 & 3 & 2 & 35 & 94 & 2 & -3 \\
\hline 8 & 110 & 30 & 40 & 1.3500 & 0.00393 & 3 & 2 & 45 & 114 & 1 & -3 \\
\hline 9 & 80 & 20 & 25 & 1.5000 & 0.00396 & 0 & 0 & 40 & 101 & 0 & -1 \\
\hline 10 & 60 & 20 & 15 & 1.4000 & 0.0051 & 0 & 0 & 30 & 85 & 0 & -1 \\
\hline
\end{tabular}

In tables 1 and 2 :

$\mathbb{P}_{\text {mas: }}$ : The maximum of generating power for each unit

$\mathbb{P}_{\text {min }}$ : The minimum generating power for units 
$\alpha, \beta$ and $\gamma$ are coefficients of cost function related to every unit which is by general form of below [15]:

$$
C=\alpha \times P^{2}+\beta \times P+\gamma
$$

MU: The minimum on mode time of each unit

MD: The minimum off mode time of each unit

Cs-hrs: is equal to the number of hours which after spending that, the required cost for restarting the unit is equal to CSC; otherwise renewed cost is equal to HSC. This can be summarized as [15]:

$$
\text { The cost of start-up unit } i=\begin{array}{ll}
\text { HSC } & x_{i}^{\text {off }} \leq M_{i}+C S_{-} \text {hrs } \\
\text { CSC } & x_{i}^{\text {off }} \geq M_{i}+C S_{-} \text {hrs }
\end{array}
$$

Where:

$x_{i}^{\text {off }}$ : The time intervals that unit $i$ was in off mode continuously.

$\mathrm{MD}_{\mathrm{j}}$ : The minimum off mode time for each unit $\mathrm{i}$

Initial state: is initial state of each unit

\subsection{Results for a system by 4 generators}

For this system in every time, we have 15 point of maximum power station mixture which for each ED has been done and its results are used as algorithm input. The UC optimization program has been developed using MATLAB code which is implemented repeatedly for 15 times. In this part, the MACO has been applied to the mentioned system in the precious subsections and the simulation results are shown in table 3

Table 3 contains 5 parts. In the first and the second columns, the number of hour and the amount of load demand at each time are mentioned respectively. In the third to sixth columns, the results of unit's ED are extracted using the UC optimization. In the seventh column the start-up costs related to each hour is expressed and the eighth column indicates the total costs related to each hour which contains the total generation costs related to the generators and the start-ups.

As indicated in table 3, the average generation cost of first generator is lower than the others and after that the second generator has the lowest cost. So firstly, generators 1 and 2 must be added to the network that most of the load will be committed by the first generator and after that as for compensation the lack of load, generator 3 should be placed in the circuit. Finally, if the required load become more than the total generated power of the first three generators, ineluctability the forth generator have to be placed in circuit.

As it is shown in table 3, the units with numbers 1 and 2 are always in circuit. Therefore, the startup costs related to these units will not appear. At the first hour, the third unit has been restarted after 4 hours of blackout according to (2)-(4) and so their start-up cost is equal to 150 . Consequently at 4th hour, only unit 4 must enter in the circuit (the others are in circuit previously); therefore, the start-up cost is only related to unit number 4 and according to (2)-(4) is equal to 0.02 dollars. For the rest of the times, the start-up costs are zero because of no new input that enters the circuit. In the last line of seventh and eighth columns the total start-up and generation costs related to all 8 hours are shown.

In table 4, obtained results of MACO are compared to the past well-known methods. In the first and second columns of this table, the planning hours and their related load demands are indicated. 
In the next columns, the different methods' results are brought. Under each method there are four numbers which show the units numbers; ' 1 ' under each unit means the on-mode and ' 0 ' means the off-mode for the unit. In the last row, the total generation costs for supplying each load are compared for different methods. As it is observed, the results of MACO are better than the obtained results from other references. And also the convergence speed and earning suitable response is favorable limitation.

\subsection{Results for a system with 10 generators}

In this system for each hour the number of power station mixture cases is very high; therefore we should limit the number of cases as much as possible which in this case the priority list has been used. For each of these cases, ED has been done and the results of ED have been used as input vector for MACO. MACO reaches the final response for a system with 10 generators fewer than 10 repeats. The results related to UC problem for a system with 10 generators is given in table 5 . In the first and second columns of table 5, the time (hour) and the related load demands are has shown. In the 3rd column (related to the units' part), there are 10 numbers from 1 to 10 which explains the number of each generator. Below the numbers, the state of each generator is placed due to each hour (' 1 ' indicates that the generator is active and ' 0 ' means its off-mode). In the last column, sum of the related costs of units are shown which contains the production and the startup costs in each hour. Either in the last row, the total costs related to all generators is brought for 24 hours.

The obtained result of MACO has compared with the results of other well-known methods as shown in table 6. In table 6, in the first and second column by order number of each hour and required load amount at that hour is mentioned. In the next columns obtained results of other methods has been brought. In under of per method there is two columns which first column is related to total required cost in that hour for generating required load and second column which shows the mode of system. In column of system modes which is in front of per hour is places by one number containing 10 digits with 0 and 1 indicates active mode or reactive mode of unit. The first digit is related to first unit and last digit is related to the last unit and number 1 means the unit is active and number 0 means it is reactive. In the last column under per method, sum of required costs for generating is shown. As we can see, obtained result of MACO by accepted limitation is better than the results of other methods and considerably decreases essential costs for required load supply.

Table 3. Obtained results of simulation by MACO for 4 units

\begin{tabular}{|c|c|c|c|c|c|c|c|}
\hline \multirow{2}{*}{ Hour } & \multirow{2}{*}{$\begin{array}{l}\text { Load } \\
\text { (MW) }\end{array}$} & \multicolumn{4}{|c|}{ Unit number } & \multirow{2}{*}{$\begin{array}{l}\text { Strat-up } \\
\text { cost }(\$)\end{array}$} & \multirow{2}{*}{$\begin{array}{c}\text { Generation } \\
\text { cost }(\$)\end{array}$} \\
\hline & & 1 & 2 & 3 & 4 & & \\
\hline 1 & 450 & 292.857 & 132.143 & 25 & 0 & 150 & 9539.038 \\
\hline 2 & 530 & 300 & 205 & 25 & 0 & 0 & 10856.240 \\
\hline 3 & 600 & 300 & 250 & 30 & 20 & 0.02 & 12534.560 \\
\hline 4 & 540 & 300 & 215 & 25 & 0 & 0 & 11043.800 \\
\hline 5 & 400 & 276.19 & 123.810 & 0 & 0 & 0 & 8205.788 \\
\hline 6 & 280 & 196.190 & 83.810 & 0 & 0 & 0 & 6067.148 \\
\hline 7 & 290 & 202.857 & 87.143 & 0 & 0 & 0 & 6243.828 \\
\hline 8 & 500 & 300 & 200 & 0 & 0 & 0 & 10030.360 \\
\hline \multicolumn{6}{|c|}{ Total } & 150.02 & 74520.344 \\
\hline
\end{tabular}

Table 4. Comparing the obtained results of simulation by MACO for 4 units with other references

\begin{tabular}{|c|c|c|c|c|c|c|c|c|c|c|c|c|c|c|c|c|c|c|c|c|c|}
\hline \multirow{2}{*}{ Hour } & \multirow{2}{*}{$\begin{array}{c}\text { Load } \\
(\mathrm{MW})\end{array}$} & \multicolumn{4}{|c|}{ МАСО } & \multicolumn{4}{|c|}{ GA [9] } & \multicolumn{4}{|c|}{ GA [10] } & \multicolumn{4}{|c|}{ PSO [14] } & \multicolumn{4}{|c|}{ ACO [23] } \\
\hline & & 1 & 2 & 3 & 4 & 1 & 2 & 3 & 4 & 1 & 2 & 3 & 4 & 1 & 2 & 3 & 4 & 1 & 2 & 3 & 4 \\
\hline 1 & 450 & 1 & 1 & 1 & 0 & 1 & 1 & 0 & 0 & 1 & 1 & 0 & 0 & 1 & 1 & 1 & 1 & 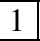 & 1 & 1 & 4 \\
\hline 2 & 530 & 1 & 1 & 1 & 0 & 1 & 1 & 0 & 0 & 1 & 1 & 1 & 0 & 1 & 1 & 1 & 0 & 1 & 1 & 1 & 0 \\
\hline 3 & 600 & 1 & 1 & 1 & 1 & 1 & 1 & 1 & 0 & 1 & 1 & 1 & 1 & 1 & 1 & 1 & 1 & 1 & 1 & 1 & 0 \\
\hline
\end{tabular}


Advanced Energy: An International Journal (AEIJ), Vol. 3, No. 2/3, July 2016

\begin{tabular}{|l|l|l|l|l|l|l|l|l|l|l|l|l|l|l|l|l|l|l|l|l|l|}
\hline 4 & 540 & 1 & 1 & 1 & 0 & 1 & 1 & 0 & 0 & 1 & 1 & 1 & 0 & 1 & 1 & 1 & 0 & 1 & 1 & 1 & 1 \\
\hline 5 & 400 & 1 & 1 & 0 & 0 & 1 & 1 & 0 & 0 & 1 & 1 & 1 & 0 & 1 & 1 & 0 & 0 & 1 & 1 & 0 & 0 \\
\hline 6 & 280 & 1 & 1 & 0 & 0 & 1 & 0 & 0 & 0 & 1 & 1 & 0 & 0 & 1 & 1 & 0 & 0 & 1 & 1 & 0 & 0 \\
\hline 7 & 290 & 1 & 1 & 0 & 0 & 1 & 0 & 0 & 0 & 1 & 1 & 0 & 0 & 1 & 1 & 0 & 0 & 1 & 1 & 0 & 0 \\
\hline 8 & 500 & 1 & 1 & 0 & 0 & 1 & 1 & 0 & 0 & 1 & 1 & 1 & 0 & 1 & 1 & 0 & 0 & 1 & 1 & 0 & 0 \\
\hline
\end{tabular}

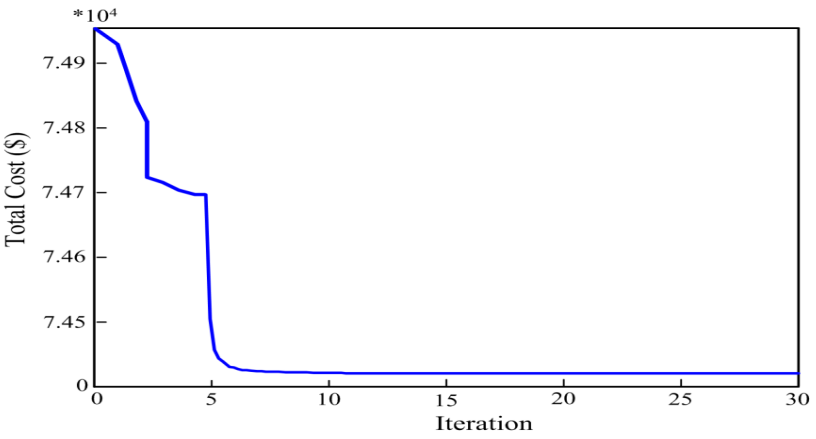

Figure 3. Speed of reaching to optimized responses for a system by 4 units

Table 5. Results of analysis for a system by 10 generators

\begin{tabular}{|c|c|c|c|c|c|c|c|c|c|c|c|c|}
\hline \multirow{2}{*}{ Hour } & \multirow{2}{*}{$\begin{array}{c}\text { Load } \\
(\mathrm{MW})\end{array}$} & \multicolumn{10}{|c|}{ Unit number } & \multirow{2}{*}{ cost $(\$)$} \\
\hline & & 1 & 2 & 3 & 4 & 5 & 6 & 7 & 8 & 9 & 10 & \\
\hline 1 & 1170 & 1 & 1 & 1 & 1 & 1 & 1 & 0 & 0 & 1 & 1 & 2433.121 \\
\hline 2 & 1250 & 1 & 1 & 1 & 1 & 1 & 1 & 1 & 1 & 0 & 1 & 1605.756 \\
\hline 3 & 1380 & 1 & 1 & 1 & 1 & 1 & 1 & 1 & 1 & 1 & 0 & 2890.637 \\
\hline 4 & 1570 & 1 & 1 & 1 & 1 & 1 & 1 & 1 & 1 & 1 & 1 & 3295.703 \\
\hline 5 & 1690 & 1 & 1 & 1 & 1 & 1 & 1 & 1 & 1 & 1 & 1 & 3578.545 \\
\hline 6 & 1820 & 1 & 1 & 1 & 1 & 1 & 1 & 1 & 1 & 1 & 1 & 3906.292 \\
\hline 7 & 1910 & 1 & 1 & 1 & 1 & 1 & 1 & 1 & 1 & 1 & 1 & 4146.285 \\
\hline 8 & 1940 & 1 & 1 & 1 & 1 & 1 & 1 & 1 & 1 & 1 & 1 & 4229.597 \\
\hline 9 & 1990 & 1 & 1 & 1 & 1 & 1 & 1 & 1 & 1 & 1 & 1 & 4378.066 \\
\hline 10 & 1990 & 1 & 1 & 1 & 1 & 1 & 1 & 1 & 1 & 1 & 1 & 4378.066 \\
\hline 11 & 1970 & 1 & 1 & 1 & 1 & 1 & 1 & 1 & 1 & 1 & 1 & 4316.945 \\
\hline 12 & 1940 & 1 & 1 & 1 & 1 & 1 & 1 & 1 & 1 & 1 & 1 & 4229.597 \\
\hline 13 & 1910 & 1 & 1 & 1 & 1 & 1 & 1 & 1 & 1 & 1 & 1 & 4146.285 \\
\hline 14 & 1830 & 1 & 1 & 1 & 1 & 1 & 1 & 1 & 1 & 1 & 1 & 3932.427 \\
\hline 15 & 1870 & 1 & 1 & 1 & 1 & 1 & 1 & 1 & 1 & 1 & 1 & 4038.283 \\
\hline 16 & 1830 & 1 & 1 & 1 & 1 & 1 & 1 & 1 & 1 & 1 & 1 & 3932.427 \\
\hline 17 & 1690 & 1 & 1 & 1 & 1 & 1 & 1 & 1 & 1 & 1 & 1 & 3578.545 \\
\hline 18 & 1510 & 1 & 1 & 1 & 1 & 1 & 1 & 1 & 1 & 1 & 1 & 3160.748 \\
\hline 19 & 1420 & 1 & 1 & 1 & 1 & 1 & 1 & 1 & 1 & 0 & 1 & 2968.299 \\
\hline 20 & 1310 & 1 & 1 & 0 & 1 & 1 & 1 & 1 & 1 & 1 & 1 & 2721.549 \\
\hline 21 & 1260 & 1 & 1 & 0 & 1 & 1 & 1 & 1 & 1 & 1 & 1 & 2614.143 \\
\hline 22 & 1210 & 1 & 1 & 0 & 1 & 1 & 1 & 1 & 1 & 1 & 1 & 2508.618 \\
\hline 23 & 1250 & 1 & 1 & 0 & 1 & 1 & 1 & 1 & 1 & 1 & 1 & 2603.263 \\
\hline 24 & 1140 & 1 & 0 & 0 & 1 & 1 & 1 & 1 & 1 & 1 & 1 & 2371.304 \\
\hline \multicolumn{12}{|c|}{ Total } & 833645.1374 \\
\hline
\end{tabular}

In figure 4 the convergence speed of MACO is depicted by considering parameters below: $\alpha=1.2 \quad \beta=20 \quad p=0.25 \quad \tau_{0}=1$

The number of ants $=200$ 
Regarding to this point is essential which giving amounts to parameters above is effective in the speed of reaching to final response and its quantity and considered parameters are regarded by trial and error. As we can see, after passing almost 25 repeats algorithm has been achieved to final response, which is in more suitable limitation rather to other methods.

\section{CONCLUSIONS}

In this paper, at first an attention is paid to explain UC problem and necessarily of existence of a suitable algorithm for solving it. Then, a modified ant colony optimization (MACO) algorithm is introduced for solving this problem and its advantages and disadvantages was perused. In continuation, after explaining the proposed algorithm and perusing its different aspects by considering the load supply constraint, spinning reserve, unit's minimum on-mode time and minimum off-mode time, the UC problem has been solved for two typical systems (one for a system with 10 generating units for 24 hours and another for a system with 4 generating units for 8 hours). Then, by doing ED, the generation costs have been calculated. Finally, they have compared to other well-known methods. The obtained simulation results show that proposed method optimized the problem more than other methods in terms of economic constraint and it is in good situation in terms of convergence speed.

Table 6. Comparing the results of simulation for a system by 10 generators with past method

\begin{tabular}{|c|c|c|c|c|c|c|c|c|c|}
\hline \multirow[t]{2}{*}{ Hour } & \multirow{2}{*}{$\begin{array}{l}\text { Load } \\
\text { (MW) }\end{array}$} & \multicolumn{2}{|c|}{ MACO } & \multicolumn{2}{|c|}{$\mathrm{ACO}[23]$} & \multicolumn{2}{|c|}{$\begin{array}{l}\text { Branch and bound } \\
{[11]}\end{array}$} & \multicolumn{2}{|c|}{$\begin{array}{c}\text { Dynamic } \\
\text { programming [4] }\end{array}$} \\
\hline & & Cost & State & Cost & State & Cost & State & Cost & State \\
\hline 1 & 170 & 2433.1 & 111111001 & 2849.6 & 1111110 & 2725.8 & 111111001 & 2638.1 & 111101001 \\
\hline 2 & 250 & 1605.7 & 11110 & 2606.6 & & 2606.1 & & 719.1 & \\
\hline 3 & 380 & 2890.6 & & 2887.0 & & 2981.7 & & 2889.4 & \\
\hline 4 & 1570 & 3295.7 & & 3396.8 & & 3409.8 & & 3426.0 & \\
\hline 5 & 1690 & 3578.5 & & 3578.7 & & 3578.7 & & 3607.4 & 101 \\
\hline 6 & 1820 & 39062 & 11 & 3906.4 & & 3906.4 & 1111 & 3948.8 & 11101 \\
\hline 7 & 1910 & 41462 & 1111 & 4246.4 & & 4146.4 & 1111 & 4247.4 & 11111 \\
\hline 8 & 1940 & 4229.5 & 111 & 4229.7 & & 4229.7 & & 4229.7 & \\
\hline 9 & 1990 & 4378.0 & 111 & 43782 & & 43782 & & 43782 & \\
\hline 10 & 1990 & 4378.0 & 111 & 43782 & 11 & 43782 & 11 & 43782 & \\
\hline 11 & 1970 & 43169 & 111 & 4317.1 & 11 & 43782 & 11 & 43782 & \\
\hline 12 & 1940 & 4229.5 & 11 & 4229.7 & & 4317.1 & & 4317.1 & \\
\hline 13 & 1910 & 41462 & & 4146.4 & & 414 & & 4146.4 & \\
\hline 14 & & 3932.4 & & 39 & & 39 & & 2.5 & \\
\hline 15 & & & & & & & & & \\
\hline 16 & & & & & & & & & \\
\hline 17 & & & & & & & & & \\
\hline 18 & & & & & & & & & \\
\hline 19 & & & & & & & & & \\
\hline 20 & & 2721.5 & & & & 2721.7 & & 27349 & 111 \\
\hline 21 & & 2614.1 & 11 & 26143 & 11 & 26143 & 11 & 26332 & 11 \\
\hline 22 & 1210 & 2508.6 & 110 & 2508.7 & 11 & 2508.7 & 11 & 25332 & 11 \\
\hline 23 & 1250 & 26032 & 111 & 2593.0 & 110 & 2593.0 & 11111 & 26129 & 111 \\
\hline 24 & 1140 & 23713 & 1111111101 & 2364.1 & 1101111111 & 2364.1 & 1101111111 & 2394.1 & 1101111111 \\
\hline \multicolumn{2}{|c|}{ Total cost } & \multicolumn{2}{|c|}{833645.1374} & \multicolumn{2}{|c|}{83491.42} & \multicolumn{2}{|c|}{834752.2} & \multicolumn{2}{|c|}{83652.4} \\
\hline
\end{tabular}




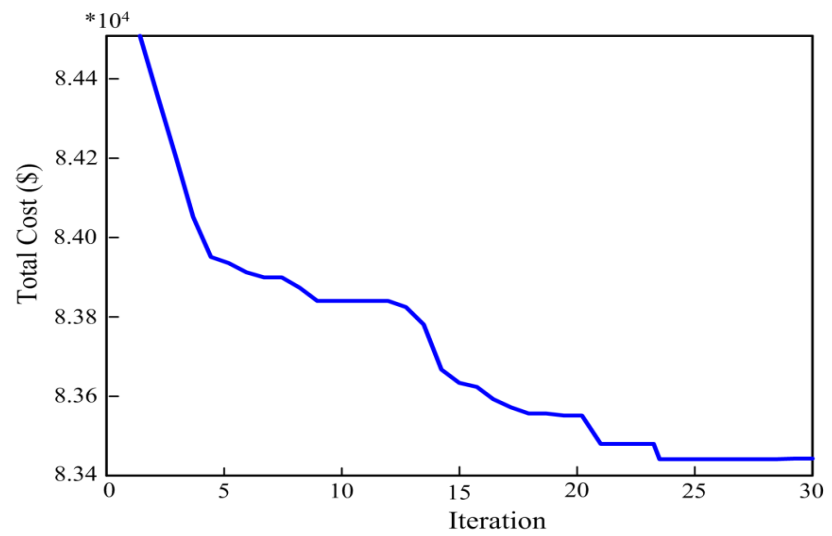

Figure 4. Reaching speed to optimized responses for a system by 10 generators

\section{REFERENCES}

[1] Wood A. F., Wollenberg B. F. Power generation operation and control. 2nd ed. New York: Wiley Press. 1998.

[2] Burns R. M., Gibson C. A. Optimization of priority lists for a unit commitment program. Proceeding of IEEE/Power Engineering Society Summer Meeting, 2007, Paper A 75 453-1.

[3] Sheble G. B. Solution of the unit commitment problem by the method of unit periods. IEEE Transaction on Power System. 1990; 5(1): 257-260.

[4] Ouyang Z., Shahidehpour S. M. An intelligent dynamic programming for unit commitment application. IEEE Transaction on Power System. 1991; 6(3): 1203-1209.

[5] Zhi C., Lili Z., Kun Z., Deyue M., Xu Z., Dan X., Sai D., Unit commitment with battery energy storage considering wind forecast error. TELKOMNIKA Indonesian Journal of Electrical Engineering. 2014; 12(5): 3357-3365.

[6] Mantawy A. H., Abdel-Magid Y. L., Selim S. Z. Unit commitment by Tabu search. IET Generation, Transmission and Distribution. 2005; 152(4): 563-574.

[7] Naama B., Bouzeboudja H., Allali A. Solving the economic dispatch problem by using Tabu search algorithm. Energy Procedia. 2013; 36(3): 694-701.

[8] Jahromi M. Z., Hosseini Bioki M. M., Rashidinejad M., Fadaeinejad R. Solution to the unit commitment problem using an artificial neural network. Turkish Journal of Electrical Engineering \& Computer Sciences. 2013; 21(2): 198-212.

[9] Zhang N., Hub Z., Hanc X., Zhanga J., Zhoua Y. A fuzzy chance-constrained program for unit commitment problem considering demand response, electric vehicle and wind power. International Journal of Electrical Power \& Energy Systems. 2015; 65(1): 201-209.

[10] Rudolf A., Bayrleithner R. A genetic algorithm for solving the unit commitment problem of a hydrothermal power systems. IEEE Transaction on Power System. 1999; 14(4); 1460-1468.

[11] Kazarlis S. A., Bakirtzis A. G., Petridis V. A genetic algorithm solution to the unit commitment problem. IEEE Transaction on Power System. 1996; 11(1): 83-92.

[12] Trivedia A., Srinivasana D., Biswasc S., Reindl T. Hybridizing genetic algorithm with differential evolution for solving the unit commitment scheduling problem. Swarm and Evolutionary Computation, under publication.

[13] Cheng C. P., Liu C. W., Liu C. C. Unit commitment by annealing-genetic algorithm. International Journal of Electrical Power \& Energy Systems. 2002; 24(2): 149-158.

[14] Yu X., Zhang X. Unit commitment using Lagrangian relaxation and particle swarm optimization. International Journal of Electrical Power \& Energy Systems. 2014; 61(6): 510-522

[15] Prateek K., Singhal R., Naresh V. S. A novel strategy-based hybrid binary artificial bee colony algorithm for unit commitment problem. Arabian Journal of Science and Engineering. 2015; 40(2): 1455-1469.

[16] Chandrasekaran K., Simon S. P. Multi-objective unit commitment problem with reliability function using fuzzified binary real coded artificial bee colony algorithm. IET Generation, Transmission \& Distribution. 2012; 6(10): 1060-1073.

[17] Govardhan M., Roy R. Economic analysis of unit commitment with distributed energy resources. Electrical Power and Energy Systems, 2015;71 (1): 1-14. 
[18] Khatibi M., Bigdeli M. Transient stability improvement of power systems by optimal sizing and allocation of resistive superconducting fault current limiters using particle swarm optimization. Advanced Energy: An International Journal (AEIJ), 2014; 1(3): 11-27.

[19] Dorigo M. Optimization, Learning and Natural Algorithms. PhD Thesis. Politecnico di Milano, Italy. 1992.

[20] Dorigo M., Stützle T. Ant Colony Optimization. MIT Press. 2004

[21] Dorigo M., Stützle T. Ant Colony Optimization: Overview and Recent Advances. Springer Verlag Press. New York. 2010.

[22] Yuan-Kang W., Chih-Cheng H., Chun-Liang L. Resolution of the unit commitment problems by using the hybrid Taguchi-ant colony system algorithm. International Journal of Electrical Power \& Energy Systems. 2013; 49(3): 188-198.

[23] Simon S. P., Padhy N. P., Anand R. S. An ant colony system approach for unit commitment problem. International Journal of Electrical Power and Energy Systems. 2006; 28(5): 315-323. 